\title{
Multifrequency observations of a sample of very low frequency peaked BL Lacertae objects
}

\author{
A. Maselli ${ }^{1}$, E. Massaro ${ }^{2}$, R. Nesci ${ }^{2}$, S. Sclavi ${ }^{3}$, C. Rossi ${ }^{2}$, and P. Giommi ${ }^{4}$ \\ 1 Istituto di Astrofisica Spaziale e Fisica Cosmica, INAF, via U. La Malfa 153, 90146 Palermo, Italy \\ e-mail: maselli@ifc.inaf.it \\ 2 Dipartimento di Fisica, Università La Sapienza, Piazzale A. Moro 2, 00185 Roma, Italy \\ 3 Stazione Astronomica di Vallinfreda, via del Tramonto, Vallinfreda (RM), Italy \\ 4 ASI Science Data Center, ASI, ESRIN, via G. Galilei, 00040 Frascati, Italy
}

Received 24 September 2009 / Accepted 16 December 2009

\section{ABSTRACT}

\begin{abstract}
Context. BL Lacertae objects with an extremely low value of the peak frequency $\left(v_{\mathrm{s}} \leq 10^{13} \mathrm{~Hz}\right)$ of the synchrotron component (VLBL objects) are poorly known.

Aims. We selected a small sample of these candidate objects on the basis of their radio/optical flux ratio to evaluate their variability, define the low energy part of their SED and obtain a reliable estimate of their $v_{\mathrm{s}}$ through a fit with a $\log$-parabolic law.

Methods. They were the object of a ground-based observational campaign in the infrared and optical bands; data from several pointings by the Swift satellite in the UV and X-ray band were also obtained for half the sample. We complemented our data with a large number of literature data.

Results. These sources showed a marked flux variability, as expected for BL Lacertae objects of the low energy peaked BL Lac objects (LBL) and intermediate BL Lac objects type; all of them have a $v_{\mathrm{s}}$ value around $10^{13} \mathrm{~Hz}$, but this is low enough only for a few sources to consider them genuine VLBL objects. We report the overall properties of individual sources and discuss some aspects of this class of rare objects, which could correspond to the transition between flat spectrum radio quasars and LBLs.
\end{abstract}

Key words. galaxies: active - BL Lacertae objects: general - radiation mechanisms: non-thermal

\section{Introduction}

Blazars are a well known class of active galactic nuclei (AGNs) which include BL Lac objects and flat spectrum radio quasars (FSRQs). Their spectral energy distributions (SED), based on data from the radio band to the highest energy $\gamma$-rays, are characterised by two main broad bumps. Following the scenario originally proposed by Blandford \& Rees (1978), the bump peaking at lower frequency is generally explained in terms of synchrotron emission from relativistic electrons which move down a jet pointing close to the observers' line of sight. The second feature, peaking at higher frequencies, is likely due to the inverse Compton upscattering of low energy photons by the same electron population. The so-called low energy peaked BL Lac objects (LBL) (Padovani \& Giommi 1995) have the maximum of the first component in the IR-optical range, where they generally show a broad and fast variability, while those peaking in the UV-X rays are named high energy peaked BL Lac objects (HBL). LBL sources observed in the X-ray band show flat spectra with an energy index $\left(S(v) \propto v^{-\alpha}\right)$ smaller than unity and mostly exhibit a rather moderate variability in this band. The SEDs of HBL sources peak as a rule above a few $\mathrm{keV}$, and their $\mathrm{X}$-ray energy indices are close to or smaller than unity. Their variability in this band is high in contrast with the optical and radio data, where their flux is more stable (Fiorucci et al. 2004).

In addition to these two classes of BL Lacs a finer taxonomy, always based on the frequency of the synchrotron peak $v_{\mathrm{s}}$, is used in the literature. Following Padovani \& Giommi (1995), sources with $\log v_{\mathrm{s}}<14.5$ (measured in $\mathrm{Hz}$ ) are LBL objects, those with $\log v_{\mathrm{s}}>16.5$ are HBLs, and those with $14.5<\log v_{\mathrm{S}}<$ 16.5 are classified as intermediate BL Lac objects (IBL). Two more classes have also been considered in the literature: the extreme HBL with $\log v_{\mathrm{s}}>18$ (Costamante et al. 2001) and the very low energy peaked BL Lacs (VLBL) with $\log v_{\mathrm{s}}<13$ (Antón \& Browne 2005). We stress that the actual distribution of $v_{\mathrm{s}}$ can be continuous and that the frequency borders of these classes, historically influenced by an observational approach, are rather arbitrary. Our aim therefore is to investigate the properties of some sources at the low-frequency end of the synchrotron peak range.

The SEDs of FSRQs are mainly similar to those of LBL objects, and only very few peculiar sources like ROXA J081009.9+384757.0 (Giommi et al. 2007) are thought to have the synchrotron peak in the UV-X range. The main difference between LBL objects and FSRQs is therefore the occurrence of broad prominent emission lines in the optical spectra. Some LBL sources in faint states occasionally showed emission lines, which modified the definition of BL Lac, allowing for lines with an equivalent width (EW) smaller than $5 \AA$ (Stickel et al. 1991). However, this limit was exceeded in some occasions by well-known sources like BL Lac itself (Vermeulen et al. 1995) and a few others that have been classified as "transition objects" between the two classes of blazars.

For a better understanding of the relationship between BL Lacs and FSRQs we decided to study the spectral properties and the variability of some VLBL candidates. The present observational knowledge of VLBL objects is poor: by definition the radio/optical flux ratio must be high for a VLBL source so 
that in a radio flux limited sample VLBLs are optically fainter than the other flavours of BL Lacs and therefore less present in optical monitoring samples. Moreover, this picture can be further complicated by considerable brightness changes often associated with an increase of the synchrotron peak frequency in the bright states. Sources potentially classified as VLBLs can therefore occasionally appear as LBLs when brighter and vice-versa.

About the nature of VLBL sources, one possibility is that they are normal LBLs with a low $v_{\mathrm{s}}$ because of a loose alignment of the jet to the line of sight, and therefore with a relatively low Doppler boosting. In this case, we expect a rather weak optical variability associated with the absence of high superluminal motions in the jet. The detection of components in the jet with a high proper motion will be useful for a reliable estimate of the beaming factor and can be used to test this interpretation.

There is no well-established and efficient way to select VLBL candidates. The best approach is of course to estimate the synchrotron peak from well-sampled spectra, possibly derived from nearly simultaneous observations. This selection criterion is however limited to a very small number of bright sources. The obvious alternative possibility is to use archive data. Nieppola et al. (2006) fitted the synchrotron peak of a very substantial sample of radio sources, reported as BL Lacs in the literature, by means of a parabola in a double-log plot (i.e. a log-normal distribution, hereafter named log-parabola) using new radio measurements combined with archive data to estimate $v_{\mathrm{s}}$. According to their results only nine of the 308 sources for which best fits were computed have $\log v_{\mathrm{s}}$ in the interval from 12.6 to 13.0. There are some problems of misclassification though, and only a few of them can be considered as genuine BL Lacs. In fact, three of these nine sources have spectra with broad lines and are FSRQs, two have a very low flux density at $1.4 \mathrm{GHz}$, with radio/optical and optical/X flux ratios similar to those of HBL sources, others are classified as blazars of an uncertain type in the Roma-BZCAT (Massaro et al. 2009). There is furthermore the possibility that peak frequencies were not always well-estimated because of the scarcity of data and of badly resulting best fits, so that some sources with $v_{\mathrm{s}}$ slightly higher than $10^{13} \mathrm{~Hz}$ are actually VLBL. In any case, apart from the classification of individual objects one can reasonably expect that VLBL sources are indeed rare objects on the basis of the existing data.

To investigate the properties of some VLBL candidates, we selected a small sample of BL Lacs and performed new optical and near IR photometry to improve the knowledge of their SED at frequencies higher than the expected $v_{\mathrm{s}}$. We present the results of our observations and estimate the peak frequency of the synchrotron component.

\section{Selection of VLBL candidates}

The major problem in the search for VLBL objects is the lack of data in the range of $10^{12}-10^{14} \mathrm{~Hz}$, which makes it difficult to estimate the $v_{\mathrm{s}}$. A first and quite simple criterion to select VLBL candidates is the assumption that the overall SED shape between radio and optical frequencies is described by a logparabola (see Sect. 5). In fact, considering that for a VLBL object $v_{\mathrm{s}}<10^{13} \mathrm{~Hz}$ and assuming for the "curvature" parameter $b$ (see Eq. (2)) a typical value in the range of $0.15-0.25$, it is easy to derive that the expected highest value of the ratio between the optical flux in the $R$ bandpass and the radio flux at $5 \mathrm{GHz}$ should be of the order of $5 \times 10^{-4}$. Thus the lowest $R$ magnitude should be $\sim 17$ for a radio flux density of $1 \mathrm{Jy}$.

Another useful quantity to classify BL Lac objects is the ratio between the X-ray and the radio flux. This ratio was used in the Sedentary Survey (Giommi et al. 2005) to select HBL objects and was found to work well also for wider BL Lac samples (Maselli 2009). In the Roma-BZCAT (Massaro et al. 2009) the X-ray flux in the $0.1-2.4 \mathrm{keV}$ band is given in units of $10^{-12} \mathrm{erg} \mathrm{cm}^{-2} \mathrm{~s}^{-1}$, while the radio flux density at $1.4 \mathrm{GHz}$ is in $\mathrm{mJy}$.

To express this ratio in a convenient adimensional number, we multiplied the radio flux density by a bandwidth of $\Delta v$, assumed equal to $1 \mathrm{GHz}$, and the value of the ratio was opportunely multiplied by a scale factor equal to $10^{-3}$

$\Phi_{X R}=10^{-3} \frac{F_{X}}{S_{1.4} \Delta v}$

In this way the value of $F_{X} / S_{1.4}=10^{-11} \mathrm{erg} \mathrm{cm}^{-2} \mathrm{~s}^{-1} \mathrm{Jy}^{-1}$ corresponds to $\Phi_{X R}=1$, a central value of the distribution that can be considered a lower limit for HBL sources (Maselli 2009). Typical values of this ratio for LBL sources are of the order of or lower than $10^{-1}$.

We selected therefore from the Roma-BZCAT a small sample of VLBL candidates in the northern sky according to the following criteria:

- BL Lac classification from a featureless optical spectrum;

$-S(5 \mathrm{GHz})>0.25 \mathrm{Jy}$;

$-V>17.0$ mag;

- X-ray to radio $(1.4 \mathrm{GHz})$ flux ratio: $\Phi_{X R}<0.1$.

A radio flux limit $S(5 \mathrm{GHz})>0.25 \mathrm{Jy}$ was adopted to have an expected optical luminosity not much fainter than $\sim 19$ mag and therefore allow an easy monitoring in the optical/NIR with $1.5 \mathrm{~m}$ class telescopes. These objects were also preferentially selected at a high north declination to observe them over a long period of the year; only one of them actually has a declination lower than $40^{\circ}$. Not all the sources satisfying these criteria could actually be observed, due to bad weather or technical problems.

The list of the eight selected sources is reported in Table 1 together with some interesting parameters taken from the literature. Throughout the paper we named the sources using the J2000 coordinates as in the Roma-BZCAT; their usual names are also given to simplify their identification. Radio fluxes of BZB J0929+8612 and BZB J1223+8040 are from the S5 survey (Kühr et al. 1981b) and for the other sources from GB6 (Gregory et al. 1996); $R$ magnitudes are from USNO-B1 (Monet et al. 2003); the reddening values are from NED; the values of $\Phi_{X R}$ (Eq. (1)) are calculated using RASS data (Voges et al. 1999); redshift values of BZB J0818+4222 and BZB J0823+2223 are from Sowards-Emmerd et al. (2005) and Stickel et al. (1993) respectively; estimates of the peak frequency and of the Doppler factor are from Nieppola et al. (2006) and Wu et al. (2007), respectively. The estimates of $v_{\mathrm{s}}$ given by Nieppola et al. (2006) are all higher than $10^{13} \mathrm{~Hz}$, although many of them are close to this threshold. We stress that our scientific goal is limited to the study of the main properties of some VLBL candidates based on a multifrequency analysis and to verify whether the resulting estimates of $v_{\mathrm{s}}$ support this classification. We also intend to check whether the adopted criteria work well for a VLBL selection to enrich the number of these relatively rare AGNs. The sample is obviously too small for statistical studies on the BL Lacs' population which require analyses of much richer and possibly complete samples.

For many of these VLBL candidates very little is known about the optical variability. They are not expected to be bright in this range: consequently, they are not included among the main targets of monitoring programmes. Nevertheless, one can expect 
A. Maselli et al.: Multifrequency observations of a sample of VLBL objects

Table 1. The list of VLBL candidates and the corresponding values of some interesting parameters taken from the literature.

\begin{tabular}{ccccccccc}
\hline \hline Source & Also known as & $\begin{array}{c}S(5 \mathrm{GHz}) \\
\mathrm{mJy}\end{array}$ & $\begin{array}{c}R \\
\mathrm{mag}\end{array}$ & $\begin{array}{c}E(B-V) \\
\mathrm{mag}\end{array}$ & $\Phi_{X R}$ & $z$ & $\log v_{\mathrm{s}}$ & $\delta$ \\
\hline BZB J0753+5352 & S4 0749+54, OI 582 & 964 & 17.5 & 0.042 & 0.052 & - & 13.12 & 9.3 \\
BZB J0818+4222 & OJ 425 & 1866 & 19.6 & 0.063 & 0.029 & $0.530 ? ?$ & 13.33 & 5.0 \\
BZB J0823+2223 & 4C 22.21, OJ 233 & 1392 & 18.4 & 0.042 & 0.007 & $0.951 ?$ & 13.09 & 2.7 \\
BZB J0832+4913 & OJ 448 & 372 & 19.2 & 0.044 & 0.044 & - & 13.06 & 8.4 \\
BZB J0929+8612 & S5 0916+86 & 253 & 19.2 & 0.154 & 0.098 & - & 14.16 & - \\
BZB J1223+8040 & S5 1221+80 & 518 & 19.3 & 0.115 & 0.027 & - & 14.21 & 4.3 \\
BZB J1927+6117 & S4 1926+61 & 558 & 17.0 & 0.065 & 0.051 & - & 13.44 & 7.7 \\
BZB J2009+7229 & S5 2010+72 & 907 & 19.0 & 0.273 & 0.020 & - & 13.64 & 7.5 \\
\hline
\end{tabular}

that they may undergo large optical flares because the emission in this band corresponds to the high frequency tail of the synchrotron jet emission. Therefore we performed an accurate photometry of archive data to derive information on their possible brightness changes over time scales longer than a decade.

\section{Observations and data reduction}

We aimed to obtain a better characterisation of the SED in the frequency range where the decay of the synchrotron component is expected for VLBLs. For this reason we carried out a groundbased photometrical campaign in the infrared and optical band for our selected BL Lacs. A posteriori we found for half of them observations by Swift (Gehrels et al. 2004) and performed the reduction of optical-UV and X-ray data acquired by the UVOT (Roming et al. 2004) and XRT (Burrows et al. 2005) telescopes aboard this mission.

\subsection{Optical-NIR observations and data reduction}

Photometric optical observations were performed in several runs from January 2005 to November 2006 at the Loiano (aperture $=152 \mathrm{~cm}$ ) and Asiago (aperture $=182 \mathrm{~cm}$ ) observatories in Italy, both equipped with liquid nitrogen-cooled CCD cameras. Standard $B$ (Johnson) and $R$ (Cousins) filters were used. Exposure times, depending upon the brightness of the source and weather conditions, were 5-10 min long for the $R$ filter and 15-20 min long for the $B$ filter to have a typical noise level on the comparison stars of 0.01 mag or less.

Bias and flat field corrections of the optical frames were performed with IRAF $^{1}$ tasks. Differential photometry with respect to some comparison stars in the same field of view of the sources was performed with the DAOPHOT task in IRAF. The same circular aperture, with a radius of $5^{\prime \prime}$, was used for the photometry of targets and of the comparison stars.

Magnitude uncertainties in the $R$ and $B$ filters were estimated from the dispersion of measurements of the reference stars in the field of each source. In each field we typically selected five stars with $B$ and $R$ magnitudes in the GSC2 catalogue. Some of them were chosen with a brightness nearly comparable to the target and some others, substantially brighter, were used to put the instrumental magnitudes from different runs on a common scale. The typical dispersion of all the reference stars was always found to be smaller than or equal to $0.05 \mathrm{mag}$, and therefore we decided to assume this value as the magnitude uncertainty for both the $R$ and $B$ filter.

\footnotetext{
1 Image Reduction and Analysis Facility, distributed by NOAO, operated by AURA, Inc. under agreement with the US NSF.
}

On three nights simultaneous observations were carried out at Campo Imperatore with the AZT24 telescope (aperture $=110 \mathrm{~cm}$ ), which is equipped with an infrared CCD liquid nitrogen cooled camera. Standard $J, H$ and $K$ filters were used; typical exposure times were about 2-3 min long. Infrared images were obtained from an appropriate stack of several short exposures performed with a locally ad hoc developed software. The $J, H$ and $K$ magnitudes for the reference stars were taken from the 2MASS catalogue. Each source was observed three times during the night, and the rms deviation from the mean was adopted as photometric error. The results of our photometry in the infrared and optical band are reported in Table 2.

\subsection{Optical-UV observations and data reduction}

The UVOT instrument (Roming et al. 2004) is able to obtain images in the $V, B, U, W 1, M 2, W 2$ filters; still, each sequence of observation may contain only some of them. The data analysis was performed with dedicated tasks included in the HEASOFT 6.6.3 package. The instrument typically takes a series of frames in each filter. We summed up each series with the UVOTIMSUM task to obtain a single frame in each filter, then performed photometry using the UVOTSOURCE task. We followed UVOT Team prescriptions (Immler et al. 2008) and adopted an aperture radius of $3^{\prime \prime}$ for the photometry, independently of the image filter. We generally took the background region in the form of an annulus around the extraction region, with inner and outer radii chosen in a way to avoid the inclusion of spurious sources, which are particularly numerous in $U V$ filter frames; typical values are $18^{\prime \prime}$ and $23^{\prime \prime}$, respectively. The obtained magnitudes were then de-reddened, using the appropriate value of $E(B-V)$ derived by Schlegel et al. (1998) and adopting the extinction curve given in Seaton (1979) with $R_{V}=3.2$, and finally converted to specific fluxes: the results are reported in Table 3.

\subsection{X-ray observations and data reduction}

The XRT observations were carried out in the most sensitive photon counting readout mode (see Hill et al. 2004, for a description of readout modes). Data were reduced with the XRTDAS software package (version 2.4.3) developed at the ASDC (ASI Science Data Center) and distributed within the HEASOFT 6.6.3 package by the NASA High Energy Astrophysics Archive Research Center (HEASARC).

We used the XIMAGE task to detect the source count rate and the centroid, and to choose a nearby source-free region to extract the background spectrum. In all the sources considered here the count rate was well below the photon pile-up threshold 
A\&A 512, A74 (2010)

Table 2. Magnitudes of VLBL candidates from our observational campaign; the error associated to the $B$ and $R$ filters is 0.05 mag.

\begin{tabular}{|c|c|c|c|c|c|c|c|c|c|}
\hline Source & Julian date & $B$ mag & $R$ mag & $J$ mag & err & $H$ mag & err & $K$ mag & err \\
\hline \multirow[t]{5}{*}{ BZB J0753+5352 } & 2453387.5 & 18.15 & 17.11 & & & & & & \\
\hline & 2453465.5 & 18.72 & 17.67 & 15.97 & 0.05 & 15.18 & 0.07 & 14.26 & 0.09 \\
\hline & 2453738.5 & 18.56 & 17.75 & & & & & & \\
\hline & 2453796.5 & 18.44 & 17.43 & & & & & & \\
\hline & 2453823.5 & 18.55 & 17.53 & & & & & & \\
\hline \multirow[t]{5}{*}{ BZB J0818+4222 } & 2453387.5 & 18.67 & 17.83 & & & & & & \\
\hline & 2453437.5 & 17.31 & 16.56 & & & & & & \\
\hline & 2453466.5 & 17.73 & 16.96 & 15.26 & 0.04 & 14.44 & 0.06 & 13.36 & 0.07 \\
\hline & 2453738.5 & 17.82 & 17.23 & & & & & & \\
\hline & 2453796.5 & 18.43 & 17.60 & & & & & & \\
\hline \multirow[t]{4}{*}{ BZB J0823+2223 } & 2453387.5 & 19.71 & 18.64 & & & & & & \\
\hline & 2453465.5 & 19.48 & 18.32 & 16.66 & 0.03 & 15.75 & 0.05 & 14.51 & 0.05 \\
\hline & 2453738.5 & 19.43 & 18.33 & & & & & & \\
\hline & 2453822.5 & 19.59 & 18.40 & & & & & & \\
\hline \multirow[t]{6}{*}{ BZB J0832+4913 } & 2453387.5 & 19.57 & 18.21 & & & & & & \\
\hline & 2453439.5 & 18.98 & 17.72 & & & & & & \\
\hline & 2453466.5 & 19.38 & 18.06 & 16.41 & 0.07 & 15.45 & 0.14 & 14.55 & 0.16 \\
\hline & 2453738.5 & 18.52 & 17.26 & & & & & & \\
\hline & 2453823.5 & 17.96 & 16.79 & & & & & & \\
\hline & 2453823.5 & & 16.77 & & & & & & \\
\hline \multirow[t]{4}{*}{ BZB J0929+8612 } & 2453557.5 & & 17.08 & & & & & & \\
\hline & 2453415.5 & 19.06 & 17.86 & & & & & & \\
\hline & 2453796.5 & 18.66 & 17.51 & & & & & & \\
\hline & 2453885.5 & 18.56 & 17.52 & & & & & & \\
\hline \multirow[t]{6}{*}{ BZB J1223+8040 } & 2453466.5 & 20.09 & & & & & & & \\
\hline & 2453466.5 & 20.04 & 18.65 & 16.85 & 0.05 & 16.06 & 0.08 & 14.71 & 0.08 \\
\hline & 2453497.5 & 19.94 & 18.55: & & & & & & \\
\hline & 2453796.5 & 21.06: & 19.40: & & & & & & \\
\hline & 2453823.5 & & 19.35 & & & & & & \\
\hline & 2453915.5 & 20.11 & 18.71 & & & & & & \\
\hline \multirow[t]{12}{*}{ BZB J1927+6117 } & 2453498.5 & 17.72 & 17.22 & & & & & & \\
\hline & 2453527.5 & 17.78 & 17.24 & 15.80 & 0.02 & 14.95 & 0.03 & 13.96 & 0.03 \\
\hline & 2453557.5 & 17.78 & 17.24 & & & & & & \\
\hline & 2453565.5 & 17.79 & 17.24 & & & & & & \\
\hline & 2453579.5 & 17.91 & 17.33 & & & & & & \\
\hline & 2453643.5 & 17.86 & 17.32 & & & & & & \\
\hline & 2453885.5 & 17.69 & 17.08 & & & & & & \\
\hline & 2453915.5 & 17.80: & 17.26 & & & & & & \\
\hline & 2453981.5 & 17.92 & 17.33 & & & & & & \\
\hline & 2453982.5 & 17.95 & 17.34 & & & & & & \\
\hline & 2454015.5 & 17.67: & 17.34 & & & & & & \\
\hline & 2454051.5 & 18.00 & 17.39 & & & & & & \\
\hline \multirow[t]{5}{*}{ BZB J2009+7229 } & 2453527.5 & & 18.87 & 17.17: & 0.03 & 15.58 & 0.03 & & \\
\hline & 2453738.5 & 20.03 & & & & & & & \\
\hline & 2453915.5 & & 19.25 & & & & & & \\
\hline & 2453981.5 & 20.51 & 19.30 & & & & & & \\
\hline & 2453982.5 & 20.71 & 19.30 & & & & & & \\
\hline
\end{tabular}

Table 3. Magnitudes of VLBL candidates observed by Swift in the six different UVOT filters.

\begin{tabular}{cccccccc}
\hline \hline Source & Date & $V$ mag & $B$ mag & $U$ mag & $W 1 \mathrm{mag}$ & $M 2 \mathrm{mag}$ & $W 2 \mathrm{mag}$ \\
\hline BZB J0753+5352 & 2006 Jan. 05 & $18.35 \pm 0.08$ & $18.93 \pm 0.08$ & $18.10 \pm 0.05$ & $18.27 \pm 0.05$ & $18.32 \pm 0.06$ & $18.50 \pm 0.04$ \\
& 2006 Jan. 06 & $18.25 \pm 0.10$ & $18.72 \pm 0.12$ & $18.04 \pm 0.07$ & $18.25 \pm 0.07$ & $18.21 \pm 0.08$ & $18.37 \pm 0.06$ \\
& 2006 Jan. 07 & $18.26 \pm 0.08$ & $18.84 \pm 0.08$ & $17.88 \pm 0.05$ & $18.26 \pm 0.06$ & $18.22 \pm 0.06$ & $18.53 \pm 0.05$ \\
\hline BZB J0818+4222 & 2006 Jan. 07 & $17.77 \pm 0.07$ & $18.28 \pm 0.05$ & $17.59 \pm 0.04$ & $17.94 \pm 0.05$ & $18.01 \pm 0.06$ & $18.30 \pm 0.05$ \\
& 2007 Jan. 18 & $18.29 \pm 0.14$ & $18.75 \pm 0.10$ & $18.03 \pm 0.09$ & $18.30 \pm 0.09$ & $>17.79$ & $18.77 \pm 0.09$ \\
& 2008 Jan. 04 & - & - & - & - & - & $18.49 \pm 0.02$ \\
& 2008 Jan. 14 & - & - & - & $17.91 \pm 0.03$ & - & - \\
& 2009 Mar. 03 & - & - & - & - & - & - \\
\hline BZB J0823+2223 & 2007 May 16 & - & - & $19.16 \pm 0.03$ & - & - & $18.96 \pm 0.02$ \\
& 2008 Oct. 17 & $18.94 \pm 0.07$ & - & $18.90 \pm 0.05$ & - & - \\
\hline BZB J2009+7229 & 2006 Feb. 17 & $>19.13$ & $>19.70$ & $>19.63$ & $>20.08$ & $>20.04$ & $>20.33$ \\
& 2006 Mar. 31 & $>19.26$ & $>20.11$ & $>19.71$ & $>20.34$ & $>20.43$ & $>20.85$ \\
\hline
\end{tabular}


Table 4. The values of the radio spectral index $\alpha_{\mathrm{r}}$, the peak frequency $v_{\mathrm{s}}$, the corresponding $v_{\mathrm{s}} S\left(v_{\mathrm{s}}\right)$ in $10^{-12} \mathrm{erg} \mathrm{cm}^{-2} \mathrm{~s}^{-1}$ and the curvature parameter $b$ characterising VLBL candidates.

\begin{tabular}{|c|c|c|c|c|c|c|c|c|c|}
\hline \multirow[b]{2}{*}{ Source } & \multirow[b]{2}{*}{$\alpha_{\mathrm{r}}$} & \multicolumn{3}{|c|}{ Faint state } & \multicolumn{5}{|c|}{ High state } \\
\hline & & $\log v_{\mathrm{s}}$ & $v_{\mathrm{S}} S\left(v_{\mathrm{S}}\right)$ & $b$ & $\alpha_{\mathrm{r}}$ & $\log v_{\mathrm{s}}$ & $v_{\mathrm{s}} S\left(v_{\mathrm{s}}\right)$ & $b$ & $\Gamma$ \\
\hline BZB J0753+5352 & -0.60 & 13.13 & 3.21 & 0.17 & -0.60 & 13.02 & 10.7 & 0.20 & $1.74 \pm 0.15$ \\
\hline BZB J0818+4222 & -0.29 & 13.15 & 3.93 & 0.21 & -0.06 & 12.75 & 9.68 & 0.19 & $1.42 \pm 0.11$ \\
\hline BZB J0823+2223 & 0.42 & 12.89 & 1.18 & 0.16 & 0.36 & 12.88 & 2.35 & 0.17 & $1.80 \pm 0.34$ \\
\hline BZB J0832+4913 & 0.11 & 12.89 & 2.61 & 0.25 & 0.02 & 13.06 & 12.5 & 0.23 & \\
\hline BZB J0929+8612 & -0.34 & 12.81 & 7.55 & 0.30 & -0.61 & 12.93 & 28.3 & 0.32 & \\
\hline BZB J1223+8040 & 0.11 & 12.97 & 2.58 & 0.20 & - & - & - & - & \\
\hline BZB J1927+6117 & -0.17 & 12.79 & 8.52 & 0.28 & - & - & - & - & \\
\hline BZB J2009+7229 & 0.07 & 13.10 & 1.06 & 0.12 & 0.07 & 12.99 & 2.57 & 0.15 & $1.96 \pm 0.33$ \\
\hline
\end{tabular}

Notes. Faint and high state are distinguished. Where available, the values of the photon index $\Gamma$ of a power law fit to Swift $\mathrm{X}$-ray data are reported.

$(\geq 0.5 \mathrm{cts} / \mathrm{s})$. Spectral data were then extracted in a circular region within a 20 pixel radius ( 1 pixel $=2^{\prime \prime} .36$ ) from the centroid; the background spectrum was estimated in a circular region of 50 pixels. We then obtained all the files necessary for the spectral analysis with the XRTPRODUCTS task; the exposure map was taken into account to correct for hot and damaged CCD pixels and for the vignetting.

In the spectral file, instrumental channels were combined to include at least 20 counts in each new energy bin. The spectral analysis was carried out in the $0.3-10 \mathrm{keV}$ energy range using XSPEC 11.3.2. Four sources of our sample have been observed by Swift and more than one observation is generally available for each of them, but only a few are provided with a suitable exposure time to produce good quality spectra. These were fitted using an absorbed power law model $N(v)=K v^{-\Gamma}$ after fixing the hydrogen column density at the Galactic value derived by Dickey \& Lockman (1990); the corresponding value of the photon index $\Gamma$ is reported in Table 4.

\section{Optical variability}

To extend the variability analysis to the longest time scales we retrieved all the scanned images available on-line from the POSS-I and POSS-II surveys and the UK-Schmidt plates in the red ( $E$ or $F$ emulsions) and blue ( $J$ emulsion) bands for each object. Differential aperture photometry on targets and reference stars for these scanned images was carried out following the same criteria described for our CCD images. Conversion from photographic magnitudes to the CCD magnitude scale was made with a calibration curve for each image by means of the reference stars; the CCD magnitude of the target was determined by interpolation with an uncertainty of $0.2 \mathrm{mag}$. The results of this photometry are reported in Table 5.

From the combined analysis of historic plates and of our two years' campaign it follows that the candidates show a marked optical variability. In particular four sources (BZB J0818+4222, BZB J0832+4913, BZB J0929+8612 and BZB J2009+7229) varied by more than $1.4 \mathrm{mag}$, and in three other cases (BZB J0753+5352 and BZB J1927+6117) the variation was confined between 0.8 and 1.4 mag; only BZB J0823+2223 had a moderate variation of 0.4 mag.

\section{Spectral energy distributions}

In the radio band the SED is generally well-described by a power law with spectral index $\alpha_{\mathrm{r}}$ up to frequencies in the range of $10^{10}-10^{11} \mathrm{~Hz}$. At higher frequencies, up to the IR-optical band, it shows a curvature well-described by a log-parabolic law that can be written as

$v S(v)=\left(v_{\mathrm{s}} S\left(v_{\mathrm{s}}\right)\right) \times 10^{-b\left(\log \left(v / v_{\mathrm{s}}\right)\right)^{2}}$,

where $v_{\mathrm{S}}$ is the frequency value at which the SED distribution reaches its peak value $v_{\mathrm{s}} S\left(v_{\mathrm{s}}\right)$ and $b$ measures the "curvature" of the parabolic model at $v_{\mathrm{s}}$. This law has the advantage to reproduce curved spectra with a small number of parameters and has been verified to fit the broad band spectra of blazars well (Landau et al. 1986; see also Massaro et al. 2004a,b, for an interpretation in terms of statistical acceleration).

The spectral energy distributions of selected VLBL objects are plotted in Fig. 1. Filled coloured symbols are our data and black open symbols are archive data; red circles mark our ground-based campaign, while blue triangles refer to POSS data; magenta squares indicate data from UVOT and XRT aboard Swift. Power law best fits in the radio and X-ray band are indicated by solid lines, while dashed lines mark the log-parabolic models of the SED. The low frequency portion of the logparabolic fit was limited to the point where it assumed the same slope of $\alpha_{\mathrm{r}}$, and we used a power law below this frequency. For sources with non-simultaneous data showing a significant variability at radio and optical frequencies, we computed the logparabolic fit to data points selecting low and high states in both ranges. A different choice, for instance that of combining a low state in the radio band with a high optical state, would introduce a bias which would increase the value of $v_{\mathrm{s}}$ and vice-versa. Bestfit parameter values for all the sources are reported in Table 4.

\section{Comments on individual sources}

For an accurate description of the synchrotron component in the SED we collected a large number of non-simultaneous multifrequency data. We took measurements from well-known catalogues, easily accessible through NED and SIMBAD databases, and integrated them with those extracted from literature articles where available. In this section we briefly describe the main properties of the sources in our sample.

\subsection{BZB J0753+5352 (OI 582)}

This source has been classified as a BL Lac object by Kühr \& Schmidt (1990) because of a featureless optical spectrum and high optical polarisation. The absence of any relevant emission feature in the optical spectrum was later confirmed by Henstock et al. (1997) and also by Stickel \& Kühr (1993), who fixed the lower limit for the redshift at $z \geq 0.2$ due to the undetection of the host galaxy. Radio data, which are available up 
Table 5. Magnitudes of VLBL candidates from our photometry on POSS-I, POSS-II and UK-Schmidt plates in the optical band.

\begin{tabular}{|c|c|c|c|c|c|c|c|c|c|}
\hline Source & Julian date & $B$ mag & $R$ mag & $J$ mag & err & $H$ mag & err & $K$ mag & err \\
\hline \multirow[t]{4}{*}{ BZB J0753+5352 } & 2434798.5 & 17.98 & 17.47 & & & & & & \\
\hline & 2435164.5 & & 16.61 & & & & & & \\
\hline & 2450896.5 & 18.43 & & & & & & & \\
\hline & 2451601.7 & & & 15.47 & 0.05 & 14.57 & 0.06 & 13.73 & 0.05 \\
\hline \multirow[t]{6}{*}{ BZB J0818+4222 } & 2435136.5 & 19.47 & 19.56: & & & & & & \\
\hline & 2447584.5 & & 17.65 & & & & & & \\
\hline & 2447860.5 & & 18.14: & & & & & & \\
\hline & 2447968.5 & 18.94 & & & & & & & \\
\hline & 2450546.5 & 18.83 & & & & & & & \\
\hline & 2450921.6 & & & 15.90 & 0.08 & 14.91 & 0.08 & 14.04 & 0.05 \\
\hline \multirow[t]{7}{*}{ BZB J0823+2223 } & 2435191.5 & & 18.64 & & & & & & \\
\hline & 2447861.5 & & 19.44: & & & & & & \\
\hline & 2449330.5 & & 19.06: & & & & & & \\
\hline & 2449751.5 & 19.79 & & & & & & & \\
\hline & 2450516.5 & 21.46: & & & & & & & \\
\hline & 2450758.5 & 21.11: & & & & & & & \\
\hline & 2450822.9 & & & 17.06: & & 16.39 & 0.23 & 15.36 & 0.12 \\
\hline \multirow[t]{4}{*}{ BZB J0832+4913 } & 2434422.5 & 19.58 & 18.29 & & & & & & \\
\hline & 2449312.5 & & 17.57 & & & & & & \\
\hline & 2450806.5 & 19.66 & & & & & & & \\
\hline & 2451595.8 & & & 16.60 & 0.15 & 15.55 & 0.15 & 14.83 & 0.10 \\
\hline \multirow[t]{14}{*}{ BZB J0929+8612 } & 2434445.5 & 19.78 & 18.67 & & & & & & \\
\hline & 2434483.5 & & 18.70 & & & & & & \\
\hline & 2447418.5 & 19.89 & & & & & & & \\
\hline & 2447418.5 & 20.00 & & & & & & & \\
\hline & 2450514.5 & 19.23 & 17.66 & & & & & & \\
\hline & 2450518.5 & & 17.95 & & & & & & \\
\hline & 2450831.5 & 19.02 & & & & & & & \\
\hline & 2450836.5 & 19.02 & & & & & & & \\
\hline & 2450841.5 & 18.81 & & & & & & & \\
\hline & 2450842.5 & & 17.54 & & & & & & \\
\hline & 2450921.5 & 19.32 & & & & & & & \\
\hline & 2450925.5 & & 17.70 & & & & & & \\
\hline & 2451161.5 & & 17.39 & & & & & & \\
\hline & 2451615.8 & & & 16.08 & 0.09 & 15.30 & 0.09 & 14.18 & 0.06 \\
\hline \multirow[t]{6}{*}{ BZB J1223+8040 } & 2435167.5 & & 18.71 & & & & & & \\
\hline & 2450467.5 & 19.66 & & & & & & & \\
\hline & 2450548.5 & 19.29 & & & & & & & \\
\hline & 2450896.5 & & 18.56 & & & & & & \\
\hline & 2450926.5 & & 18.62 & & & & & & \\
\hline & 2451300.6 & & & 16.36 & 0.11 & 15.32 & 0.10 & 14.42 & 0.07 \\
\hline \multirow[t]{5}{*}{ BZB J1927+6117 } & 2434237.5 & 17.90 & 17.30 & & & & & & \\
\hline & 2434650.5 & 17.52 & 16.82 & & & & & & \\
\hline & 2447733.5 & 17.22 & & & & & & & \\
\hline & 2448501.5 & & 16.71 & & & & & & \\
\hline & 2451320.9 & & & 15.54 & 0.05 & 14.77 & 0.05 & 13.89 & 0.06 \\
\hline \multirow[t]{4}{*}{ BZB J2009+7229 } & 2434626.5 & & 18.76 & & & & & & \\
\hline & 2448476.5 & & 19.20 & & & & & & \\
\hline & 2449189.5 & & 19.52 & & & & & & \\
\hline & 2449931.5 & & 20.29 & & & & & & \\
\hline
\end{tabular}

Notes. the error associated to measures in the $B$ and $R$ filters is 0.20 mag. In the near-infrared band, magnitudes from 2MASS are also reported.

to $37 \mathrm{GHz}$ (Teräsranta et al. 2005; Nieppola et al. 2007), evidence a very fast rising of the synchrotron emission component ( $\alpha=-0.60)$. The source was detected by ROSAT and is included both in the Faint Source Catalog (FSC, Voges et al. 2000) and in the RGB sample (Laurent-Muehleisen et al. 1999). Reich et al. (2000) reported an integrated flux density of $(9.66 \pm 2.99) \times$ $10^{-13} \mathrm{erg} \mathrm{cm}^{-2} \mathrm{~s}^{-1}$ in the $0.1-2.4 \mathrm{keV}$ band, with a power law photon index $\Gamma=(2.9 \pm 0.8)$. Three recent observations, separated by a very short time interval, have been carried out by Swift -XRT: the corresponding X-ray spectra show a very similar behaviour, and we reported in the SED only data relative to the observation with the higher statistics. Our optical data indicate a moderate variability, and the two best fits of the faint and high state (Fig. 1) are rather similar with $v_{\mathrm{S}}$ higher than $10^{13} \mathrm{~Hz}$ : therefore it cannot be properly considered a VLBL object.

\subsection{BZB J0818+4222 (OJ 425)}

This source, observed in the radio and submillimetric band up to $230 \mathrm{GHz}$ (Steppe et al. 1992) shows a flat radio spectrum with 
A. Maselli et al.: Multifrequency observations of a sample of VLBL objects
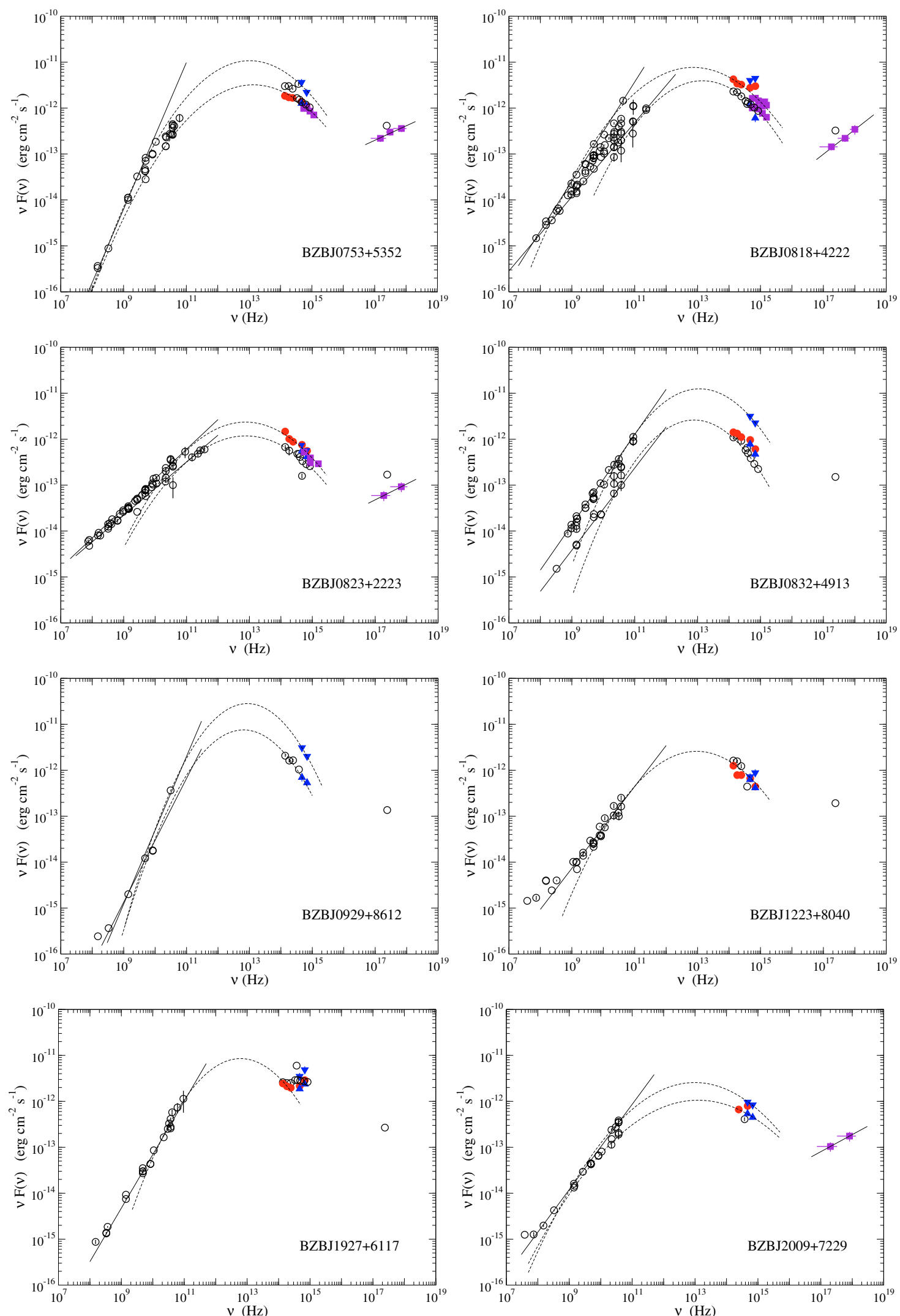

Fig. 1. Spectral energy distributions of selected VLBL sources. Power law best fits in the radio and X-ray band are indicated by solid lines, while dashed lines mark the log-parabolic models of the SED.

a flux density at $5 \mathrm{GHz}$ of about $3.3 \mathrm{Jy}$ (Kühr et al. 1981a). The combined analysis of data from the POSS plates and our campaign indicates a variability range of more than two magnitudes.
Available optical spectra (Stickel et al. 1993; Lawrence et al. 1996; Rector \& Stocke 2001; SDSS-DR6) are featureless and redshift estimates are very uncertain because the host galaxy is 
undetected, suggesting the lower limit to be $z \geq 0.6$ (Falomo et al. 1997). Its VLBL classification is controversial: we computed two SED best fits for two brightness states but excluded the high flaring values observed by us (Fig. 1), and we found the $v_{\mathrm{S}}$ value in the low state to exceed $10^{13} \mathrm{~Hz}$, while it was below this threshold in the high state. Such a behaviour (redder when brighter) is in contrast with other well studied LBL objects, but we cannot exclude that this could be due to data selection. BZB J0818+4222 was detected in the X-ray band by ROSAT (Urry et al. 1996) with $1 \mathrm{keV}$ flux of $0.05 \mu \mathrm{Jy}$, but the spectral slope is very uncertain with a photon index of $1.16_{-0.90}^{+0.67}$ in the energy range of $0.1-2.0 \mathrm{keV}$. The recent observation by Swift XRT reported here caught this source in a somewhat higher state, with a flux of $0.07 \mu \mathrm{Jy}$ and a steeper spectral index in the $0.3-10 \mathrm{keV}$ energy range.

\subsection{BZB J0823+2223 (OJ 233)}

It is a bright radio source with a rather unusual radio structure (Gabuzda et al. 2001). Its uncertain high redshift $(z=0.951)$ was estimated from a possible MgII emission line (Stickel et al. 1993). The results of our photometry confirm a low brightness level. Considering also archive values, they point out a mild variability of about $0.4 \mathrm{mag}$. The radio spectrum is the steepest one, and one cannot exclude that this value is due to the presence of some extended emission regions, as shown by the above quoted radio images. Both $v_{\mathrm{s}}$ values are very similar and lower than $10^{13} \mathrm{~Hz}$ : for this reason this source can be considered an interesting VLBL candidate.

\subsection{BZB J0832+4913 (OJ 448)}

The redshift $z=0.548$ was estimated from a possible [OIII] emission line (Stickel et al. 1993). A large variability in the optical band of about two magnitudes was reported by Walsh et al. (1984), who found it at a magnitude of $R \simeq 16.9$ mag. Our observations confirmed such a bright state with a variation range of $\sim 1.7 \mathrm{mag}$, whereas the SDSS photometry corresponds to a much fainter state. Radio data allow us to establish two rather well-defined brightness states with similar spectral indices, and therefore we computed best fits for both conditions (Fig. 1). Spectral curvatures were similar, but the peak frequency in the high state is above our VLBL threshold: consequently, this source does not properly belong to this class of BL Lacs, particularly if the high $z$ value is confirmed by new spectroscopic data.

\subsection{BZB J0929+8612 (S5 0916+86)}

Literature data in the radio band are few and quite scattered, and our photometry is limited only to the optical band. Consequently, the spectrum cannot be well-constrained at low frequencies and the log-parabolic best fits of the SED can be more uncertain than for the other sources. The resulting $v_{\mathrm{S}}$ are always lower than $10^{13} \mathrm{~Hz}$, and the high value of $v_{\mathrm{s}}$ given by Nieppola et al. (2006) is not confirmed. This source can be considered as a good VLBL candidate.

\subsection{BZB J1223+8040 (S5 1221+80)}

A featureless optical spectrum has been reported by Henstock et al. (1997). Below $\sim 1 \mathrm{GHz}$ the spectrum is steeper than at higher frequencies: this can be considered as an indication of more components with a spectrum different from the radio core, likely the dominant structure at high frequencies. For these reasons, radio data at frequencies lower than $4 \times 10^{8} \mathrm{~Hz}$ were not included in the fit. We observed this source at a few epochs and found an indication for a variability of about one magnitude, but the photometric accuracy in the faint state is rather poor. Values of $v_{\mathrm{s}}$ higher than $10^{14} \mathrm{~Hz}$, as derived by Nieppola et al. (2006), have not been found by us: the peak frequency of the unique best fit (Fig. 1) is slightly lower than $10^{13} \mathrm{~Hz}$, hence this source could be a VLBL object, but this needs to be confirmed by other data, possibly in different brightness states.

\subsection{BZB J1927+6117 (S4 1926+61)}

This source was classified as a BL Lac object by Kühr \& Schmidt (1990). The absence of detectable features both in emission and in absorption was later confirmed by all subsequent spectra. No host galaxy has ever been evidenced, suggesting $z>0.2$. The radio spectrum is very flat across a large frequency range, which makes it detectable in the microwave range by WMAP (Bennett et al. 2003). In the $\mathrm{X}$ rays, Britzen et al. (2007) reported a flux of $(0.58 \pm 0.13) \times 10^{-12} \mathrm{erg} \mathrm{cm}^{-2} \mathrm{~s}^{-1}$ in the $0.1-2.4 \mathrm{keV}$ range. We observed it on several occasions and found a rather stable brightness with a variation range within 0.3 mag. However, it was characterised by a faint $B-R$ colour, which implies a flat optical spectrum, which in turn does not follow the log-parabolic law connecting radio points. A similar flat spectral distribution follows from SDSS photometry. Assuming that the log-parabola must fit the $K$ flux (Fig. 1) we obtained a value of the curvature parameter higher than the other sources and a peak frequency below the VLBL limit.

\subsection{BZB J2009+7229 (S5 2010+72)}

This source was generally very faint, and only on a few occasions its $R$ magnitude was brighter than 19 mag. It was undetected in all the bandpasses of Swift-UVOT, and archive data indicate a comparable luminosity level, considering their large uncertainties. Two possible SEDs are shown in Fig. 1 with peak frequencies around the limit value of $10^{13} \mathrm{~Hz}$. It is therefore a possible VLBL candidate, but more data in different luminosity states are necessary to confirm its classification.

\section{Discussion}

We have presented new observational results covering the frequency interval from the near infrared to the X-ray band of a small sample of BL Lacs, which were selected to be VLBL candidates. We have also considered a collection of archive data useful to complete the description of their spectral energy distributions and, more specifically, to obtain a good estimate of the peak frequencies of the synchrotron component.

The analysis of recent Swift-XRT observations of four sources of the sample (one of them, BZB J2009+7229, was not included in the RASS) confirms that the optical/X-ray ratios and the X-ray spectral indices are similar to those expected from LBL sources. This result supports that the X-ray emission can be explained by inverse Compton scattering in the framework of synchrotron-self Compton models.

Optical monitoring and comparison with historic data show that all our candidate VLBL sources have a significant variability comparable with LBL objects: this implies that a jet disalignment with respect to the line of sight should not be the most likely explanation for their high radio/optical flux ratio. 
Table 6. Absolute magnitudes $M_{R}$ of sample sources calculated adopting $R 1$ values from USNO-B1 (Table 1 ) and rest-frame peak frequencies $v_{\mathrm{s}}$ adopting values relative to the faint state, as reported in Table 4.

\begin{tabular}{cccc}
\hline \hline Source & $m-M$ & $M_{R}$ & $v_{\mathrm{s}}$ \\
\hline BZB J0753+5352 & -42.3 & -24.8 & 13.28 \\
BZB J0818+4222 & -43.0 & -23.3 & 13.33 \\
BZB J0823+2223 & -44.6 & -25.9 & 13.18 \\
BZB J0832+4913 & -42.3 & -23.1 & 13.04 \\
BZB J0929+8612 & -42.3 & -23.1 & 12.96 \\
BZB J1223+8040 & -42.3 & -24.0 & 13.12 \\
BZB J1927+6117 & -42.3 & -25.3 & 12.94 \\
BZB J2009+7229 & -42.3 & -23.3 & 13.25 \\
\hline
\end{tabular}

Out of our eight candidates, four showed a $v_{\mathrm{s}}$ below $10^{13} \mathrm{~Hz}$, supporting their identification as VLBL sources. For three other sources the peak frequency was found in one observation lower and in the other one slightly higher than this limit; only one source was found (slightly) above it. The selection criteria adopted to build our sample have therefore a satisfactory efficiency. We cannot identify any systematic trend of $v_{\mathrm{s}}$ with the brightness level of the sources.

An important point to be taken into account is that the redshift for the majority of our sources is unknown and that the few estimated values (see Table 1) are relatively high for BL Lac objects. The distribution of known $z$ for the BL Lacs in the RomaBZCAT (Massaro et al. 2009) peaks around 0.3 and decays fast for higher values. If the typical redshifts of our sources were actually higher than 0.3 , their rest-frame frequencies $v_{\mathrm{s}}$ would result close to or higher than the VLBL limit.

We calculated the absolute magnitudes of the sources included in our VLBL sample adopting the $R$ magnitudes from USNO-B1 and assuming $z=0.4$ for all sources with unknown redshift. To compare our results with the study of the wellknown sample of host galaxies of BL Lac objects with the Hubble Space Telescope (Urry et al. 2000), we also assumed $H_{0}=50 \mathrm{~km} \mathrm{~s}^{-1} \mathrm{Mpc}^{-1}$ and $q_{0}=0$. With this comparison we expect that the absolute magnitudes of our sources are substantially brighter than the typical host galaxy. Two points seem remarkable from the results which are reported in Table 6: i) there is no clear correlation between $v_{\mathrm{s}}$ and $M_{R}$; ii) four sources have an $M_{R}$ substantially fainter than -23.7 , the typical absolute magnitude of the host galaxies of BL Lacs derived by Urry et al. (2000). This second point may imply either that the actual redshifts of our sources are even higher than 0.4 , in which case the rest-frame $v_{\mathrm{s}}$ would be higher than $10^{13} \mathrm{~Hz}$, or that we are dealing with sources with a below-the-average host galaxy. This could be related to the low peak frequency of these AGNs, but a more substantial sample of sources with known redshifts would be necessary to shed light on this topic.

A comparison between the absolute luminosity of our VLBL candidates and FSRQs is difficult from a statistical point of view due to the lack of actual redshift information for most of our sources and to the observational biases present in the AGN samples. Assuming the radio/optical flux ratio $F_{\mathrm{R}} / F_{\mathrm{O}}$ as a proxy of $v_{\mathrm{S}}$, we selected from the Roma-BZCAT a sample of 150 FSRQs with $z<1$ and $F_{\mathrm{R}} / F_{\mathrm{O}} \geq 2 \times 10^{3}$; radio fluxes were taken from NVSS (Condon et al. 1998) and R1 optical magnitudes from USNO-B1. The median value of FSRQs $\left\langle M_{R}\right\rangle=-24.9$ was found to be brighter than that of our small sample $\left\langle M_{R}^{\star}\right\rangle=-23.7$; still, one has to take into account that the rms scatter of the former sample $\left(\sigma_{M}=1.4 \mathrm{mag}\right)$ is quite high.
Wu et al. (2007) and Nieppola et al. (2008) argued that the Doppler factor $\delta$ in AGNs is anticorrelated with $v_{\mathrm{s}}$, albeit a large scatter: on average, QSOs are more boosted than LBLs, which in turn are more boosted than HBLs. Therefore one would expect that VLBL sources have the highest Doppler factors among BL Lacs and could be the transition case between BL Lacs and FSRQs. According to the measurements reported in Hovatta et al. (2009, their Table 2) the median Doppler boosting factor for BL Lac Objects is $\langle\delta\rangle=6.25$. We found an estimate of this factor for seven of our sources in Wu et al. (2007). These were derived from the correlation between the core and the total lowfrequency radio power for a sample of FRI galaxies (Giovannini et al. 2001) which holds also for a sample of BL Lacs as shown by Giroletti et al. (2004). We reported these estimates in Table 1: the median value $\left\langle\delta^{\star}\right\rangle=7.5$ agrees reasonably well with expectations, given the possible biases intrinsic to the method and the small size of the sample. The evolutionary model of blazars proposed by Cavaliere \& D'Elia (2001) considers BL Lacs as FSRQs in which the broad line region is depleted. In the hypothesis that VLBLs are FSRQs approaching the last phase of depletion we would expect a median redshift moderately higher than BL Lac's and a Doppler factor lower than FSRQ's, consistent with our findings. Clearly a larger and better defined statistical sample will be necessary to confirm these indications.

Very useful for a reliable identification of a population of VLBL sources will be the data obtained with the recent PLANCK and HERSCHEL space observatories. These instruments will in fact cover the frequency range where the SED peak frequency of VLBLs is expected, and therefore their data will for the first time provide a direct information on it. Finally we stress that one cannot exclude that a weak $\gamma$-ray emission from some VLBL candidates could also be detected in the coming years by the LAT instrument on board Fermi-GST. A detection will help to establish the nature of these AGNs.

\section{Conclusions}

The main results of this work can be summarised as follows:

- VLBL sources are very rare in blazar catalogues: this can be due to observational selection effects, classification issues or a real scarcity of sources;

- we selected a small sample of VLBL candidates from radio/optical fluxes and made an optical-NIR and NUV-X-ray monitoring;

- the variability behaviour of the sources was similar to that of normal LBL objects;

- the peak frequency of the synchrotron emission $v_{\mathrm{S}}$ of our sample objects is below or very close to $10^{13} \mathrm{~Hz}$, as expected from our selection criteria, confirming their VLBL nature;

- the redshifts of most of our sources are unknown but they must be higher than the typical values of classified BL Lacs, if their host galaxies are similar to those of the known BL Lacs;

- if the anticorrelation between $v_{\mathrm{s}}$ and Doppler beaming holds for VLBLs, these may be transition objects between FSRQ and LBL; in this scenario a relatively high redshift would naturally be expected for these sources; a fast transition could explain their rarity;

- given the large cosmic scatter of the above correlation, a number of low-power VLBL sources may still be hidden in poorly studied radio galaxies.

Acknowledgements. We thank the referee Elina Nieppola for constructive criticism which helped to improve the paper. This research has made use of 
the NASA/IPAC Extragalactic Database (NED), which is operated by the Jet Propulsion Laboratory, California Institute of Technology, under contract with the National Aeronautics and Space Administration, and of the SIMBAD database which is operated at CDS, Strasbourg, France. The authors acknowledge the financial support from "Sapienza" - Università di Roma.

\section{References}

Antón, S., \& Browne, I. W. A. 2005, MNRAS, 356, 225

Bennett, C. L., Hill, R. S., Hinshaw, G., et al. 2003, ApJS, 148, 97

Blandford, R. D., \& Rees, M. J. 1978, Proceedings of Pittsburgh Conference on BL Lac Objects

Britzen, S., Brinkmann, W., Campbell, R.M. et al., 2007, A\&A, 476, 759

Burrows, D. N., Hill, J. E., Nousek, J. A., et al. 2005, Space Sci. Rev., 120, 165

Cavaliere, A., \& D'Elia, V. 2002, ApJ, 571, 226

Condon, J. J., Cotton, W. D., Greisen, E. W., et al. 1998, AJ, 115, 1693

Costamante, L., Ghisellini, G., Giommi, P., et al. 2001, A\&A, 371, 512

Dickey, J., \& Lockman, F. 1990, ARA\&A, 28, 215

Falomo, R., Urry, M. C., Pesce, J. E., et al. 1997, ApJ, 476, 113

Fiorucci, M., Ciprini, S., \& Tosti, G. 2004, A\&A, 419, 25

Gabuzda, D. C., Pushkarev, A. B., \& Garnich, N. N. 2001, MNRAS, 327, 1

Gehrels, N., Chincarini, G., Giommi, P. et al., 2004, ApJ, 611, 1005

Giommi, P., Piranomonte, S., Perri, M. \& Padovani, P., 2005, A\&A, 434, 385

Giommi, P., Massaro, E., Padovani, P. et al., 2007, A\&A, 468, 97

Giovannini, G., Cotton, W. D., Feretti, L., Lara, L., \& Venturi, T. 2001, ApJ, 552, 508

Giroletti, M., Giovannini, G., Taylor, G. B., \& Falomo, R. 2004, ApJ, 613, 752

Gregory, P. C., Scott, W. K., Douglas, K., \& Condon, J. J. 1996, ApJS, 103, 427 Henstock, D. R., Browne, I. W. A., Wilkinson, P. N., \& McMahon, R. G. 1997, MNRAS, 290, 380

Hill, J. E., Burrows, D. N., Nousek, J. A., et al. 2004, Proc. SPIE, 5165, 217

Hovatta, T., Valtaoja, E., Tornikoski, M., \& Lähteenmäki, A. 2009, A\&A, 498, 723

Immler, S., et al. 2006, The Swift -UVOT Software guide, version 2.2

Kühr, H., \& Schmidt, G. D. 1990, AJ, 99, 1

Kühr, H., Witzel, A., Pauliny-Toth, I. I. K., \& Nauber, U. 1981a, A\&AS, 45, 367

Kühr, H., Pauliny-Toth, I. I. K., Witzel, A., \& Schmidt, J. 1981b, AJ, 86, 6
Lamer, G., Brunner, H., \& Staubert, R. 1996, A\&A, 311, 384 Landau, R., Golisch, B., Jones, T. J. et al., 1986, ApJ, 308, 78 Laurent-Muehleisen, S. A., Kollgaard, R. I., Feigelson, E. D., et al. 1999, ApJ, 525,127

Lawrence, C. R., Zucker, J. R., Readhead, A. C. S., et al. 1996, ApJS, 107, 541

Marchã, M. J., Caccianiga, A., Browne, I. W. A., \& Jackson, N. 2001, MNRAS, 326,1455

Maselli, A. 2009, Ph.D. Thesis, Sapienza, Università di Roma

Massaro, E., Perri, M., Giommi, P., \& Nesci, R. 2004a, A\&A, 413, 489

Massaro, E., Perri, M., Giommi, P., et al. 2004b, A\&A, 422, 103

Massaro, E., Giommi, P., Leto, C. et al. 2009, A\&A, 495, 691 (http://www.asdc.asi.it/bzcat)

Monet, D. G., Levine, S. E., Canzian, B., et al. 2003, AJ, 125, 984

Nieppola, E., Tornikoski, M., \& Valtaoja, E. 2006, A\&A, 445, 441

Nieppola, E., Tornikoski, M., Lähteenmäki, A., et al. 2007, AJ, 133, 1947

Nieppola, E., Valtaoja, E., Tornikoski, M., et al. 2008, A\&A, 488, 867

Padovani, P., \& Giommi, P. 1995, ApJ, 444, 567

Rector, T. A., \& Stocke, J. T. 2001, AJ, 122, 565

Reich, W., Fürst, E., Reich, P., et al. 2000, A\&A, 363, 141

Roming, P. W. A., Hunsberger, S. D., Mason, K. O., et al. 2004, in X-Ray and Gamma-Ray Instrumentation for Astronomy XIII, Proc. SPIE, 5165, 262

Schlegel, D. J., Finkbeiner, D. P., \& Davis, M. 1998, ApJ, 500, 525

Seaton, M. J. 1979, MNRAS, 187, 73

Sowards-Emmerd, D., Romani, R. W., Michelson, P. F., et al. 2005, ApJ, 626, 95

Steppe, H., Liechti, S., Mauersberger, R., et al. 1992, A\&AS, 96, 441

Stickel, M., \& Kühr, H. 1993, A\&AS, 101, 521

Stickel, M., Fried, J. W., \& Kühr, H. 1989, A\&AS, 80, 103

Stickel, M., Padovani, P., Urry, C. M., et al. 1991, ApJ, 374, 431

Stickel, M., Fried, J. W., \& Kühr, H. 1993, A\&AS, 98, 393

Teräsranta, H., Wiren, S., Koivisto, P., et al. 2005, A\&A, 440, 409

Urry, M. C., Sambruna, R. M., Worrall, D. M., et al. 1996, ApJ, 463, 424

Urry, M. C., Scarpa, R., O’Dowd, M., et al. 2000, ApJ, 532, 816

Vermeulen, R. C., Ogle, P. M., Tran, H. D., et al. 1995, ApJ, 452, L5

Voges, W., Aschenbach, B., Boller, T., et al. 1999, A\&A, 349, 389

Voges, W., Aschenbach, B., Boller, T., et al. 2000, IAUC, 7432, 1

Walsh, D., Beckers, J. M., Carswell, R. F., \& Weymann, R. J. 1984, MNRAS, 211,105

Wu, Z., Jiang, D. R., Gu, M., \& Liu, Y. 2007, A\&A, 466, 63 